\title{
The visual word form area: expertise for reading in the fusiform gyrus
}

\author{
Bruce D. McCandliss ${ }^{1}$, Laurent Cohen $^{2,3}$ and Stanislas Dehaene ${ }^{3}$ \\ ${ }^{1}$ Sackler Institute for Developmental Psychobiology, Weill Medical College of Cornell University, Box 140, 1300 York Avenue, \\ New York, NY 10021, USA \\ ${ }^{2}$ Institut de Neurologie, IFR 49, Hôpital de la Salpêtrière, AP-HP, Paris, France \\ ${ }^{3}$ INSERM unit 562 'Cognitive Neuroimaging', Service Hospitalier Frédéric Joliot, IFR 49, CEA/DRM/DSV, Orsay, France
}

\begin{abstract}
Brain imaging studies reliably localize a region of visual cortex that is especially responsive to visual words. This brain specialization is essential to rapid reading ability because it enhances perception of words by becoming specifically tuned to recurring properties of a writing system. The origin of this specialization poses a challenge for evolutionary accounts involving innate mechanisms for functional brain organization. We propose an alternative account, based on studies of other forms of visual expertise (i.e. bird and car experts) that lead to functional reorganization. We argue that the interplay between the unique demands of word reading and the structural constraints of the visual system lead to the emergence of the Visual Word Form Area.
\end{abstract}

Years after children first learn to decode letters into words, a form of perceptual expertise emerges in which groups of letters are rapidly and effortlessly conjoined into integrated visual percepts - a process that is crucial to fluent reading ability. This article reviews the current evidence in support of a link between visual word form perception and a functional specialization in a brain region that we propose to call the 'Visual Word Form Area' (VWFA) - a portion of the left fusiform gyrus that is particularly responsive to visual words. Systematic exploration of the response properties of this region reveals sensitivity to specific and abstract qualities of visual word forms that are not easily attributable to more basic stimulus properties, and are also separable from higher-order linguistic properties.

Considering the origin of VWFA poses a paradox for evolutionary accounts of functional specialization of extrastriate visual areas, given that the cultural invention of writing upon which this specialization is predicated did not exist until $\sim 5400$ years ago, which is insufficient time for selective pressures to engineer a specialized 'module' for visual word recognition. Instead, we suggest a developmental process through which reading experience drives progressive specialization of a pre-existing inferotemporal pathway for visual object recognition. Under this view, the rise of perceptual expertise in word recognition provides a remarkable example of how

Corresponding author: Bruce D. McCandliss (bdm2001@med.cornell.edu). specialization processes within the visual system can accommodate a novel cultural invention.

Perceptual expertise underlying visual word recognition Literate adults possess a special form of visual expertise that allows their visual system to process words efficiently. Within less than $250 \mathrm{~ms}$ of viewing a written word, the visual system extracts the information needed to identify its linguistic significance, despite wide variations in print, script, font, size and retinal position. Several lines of cognitive evidence indicate that the perceptual mechanisms that support word recognition rest on a critical process that groups the letters of a word together into an integrated perceptual unit (i.e. a 'visual word form'). This process is highly sensitive to the abstract structural properties of the writing system, but largely insensitive to non-essential variations in visual features such as case and font [1]. Chronometric studies show that the speed of word recognition is remarkably uninfluenced by the number of letters present in a 3-6-letter word, indicating a form of parallel processing of the letters [2]. Furthermore, the 'word superiority effect' demonstrates that a single target letter is identified more efficiently in the context of a well-structured word than within a randomly structured letter string or a letter in isolation [3]. Similar enhancement effects for letter perception have been found within meaningless letter strings that are merely consistent with the structural constraints of the writing system (i.e. pseudowords) [4,5], suggesting that the processes that support word superiority effects are prelexical and generalize readily to novel stimuli. Finally, these perceptual operations adapt well to large variations in surface features of word stimuli, such as changes in size, case, and font, and demonstrate significant transfer of priming benefits across such variations [1]. Together, these effects support the existence of a level of perceptual processing that extracts invariant information about the structure of visual words and integrates this information into a perceptual object.

We propose that a large body of converging evidence from neuroimaging studies now exists to link the psychological process of abstract visual word form perception with a particular cortical region within the left fusiform gyrus. Throughout this paper, we refer to this region as the Visual Word Form Area (VWFA). Note that 
this terminology need not imply that this patch of cortex is solely dedicated to word recognition, any more than it suggests that downstream regions are insensitive to similar stimulus contrasts. There is ample evidence that object and face recognition can also activate this region to varying degrees [6], and that activity in other regions can be modulated by presentations of visual word forms. Nevertheless, we argue that this region of the visual system must contain a population of neurons that, as an ensemble, is tuned to invariant stimulus properties and structural regularities characteristic of written words.

\section{Localizing the VWFA \\ Spatial localization}

Numerous neuroimaging studies have provided converging evidence in support of a central finding: Perception of visual words and pseudowords reliably activates the left fusiform gyrus to a greater degree than other highly similar control stimuli. Although early neuroimaging efforts produced various estimates for the localization of visual word form activity (for a review, see [7]), more recent experiments demonstrate reliable activation for words, versus stimuli that control for visual stimulation, in clusters within the left occipitotemporal sulcus bordering the fusiform gyrus. Based on reviews of over 25 group experiments, the peak of the visual word form area is located at approximately $\mathrm{x}=-43, \mathrm{y}=-54, \mathrm{z}=-12$ $(\mathrm{mm}$, to the left, posterior, and below the anterior commissure, respectively) [8]. This finding is robust enough to identify a significant peak of activation in over $90 \%$ of individual subject scans, with a standard deviation of $\sim 5 \mathrm{~mm}$ [8-11] (Fig. 1).

\section{Localization in time}

Another important class of evidence that links this region to the cognitive process of visual word form perception lies in the shared time-course between perception and activation. Consistent with the rapid time course of visual word recognition, event-related potentials $(\mathrm{ERPs})$ recorded over left posterior visual regions within $150-200 \mathrm{~ms}$ demonstrate sensitivity to words relative to other stimuli that control for visual features $[12,13]$. Similar results have also been found with magneto-encephalography (MEG) [14,15] and intracranial electrical recordings [16] that provide increasingly specific localization information that is consistent with the fMRI findings for the VWFA.

\section{Response properties of the VWFA}

Now that this region can be reliably identified and repeatedly probed under different experimental contrasts, it is possible to address questions concerning the nature of the associated cognitive operations by investigating the response properties of this region. Such research has demonstrated several fundamental characteristics of the response properties of the VWFA that are relevant for establishing its operational role in visual word perception.

\section{Modality specificity}

We argue that the VWFA corresponds to a specific visual process, rather than a more linguistic multimodal one. Passive presentation of spoken words elicits no response in this region [11]. Although nearby regions are active in word tasks that involve nonvisual modalities [17], such regions are typically significantly more anterior than the VWFA [8]. Finally, converging neuropsychological evidence indicates that selective damage to the left fusiform region impairs visual but not auditory word recognition (Box 1).

\section{Activation without awareness}

The VWFA produces measurable responses to words, even under conditions that prevent awareness of the visual presentation of a word [18] (Fig. 2). Such effects of

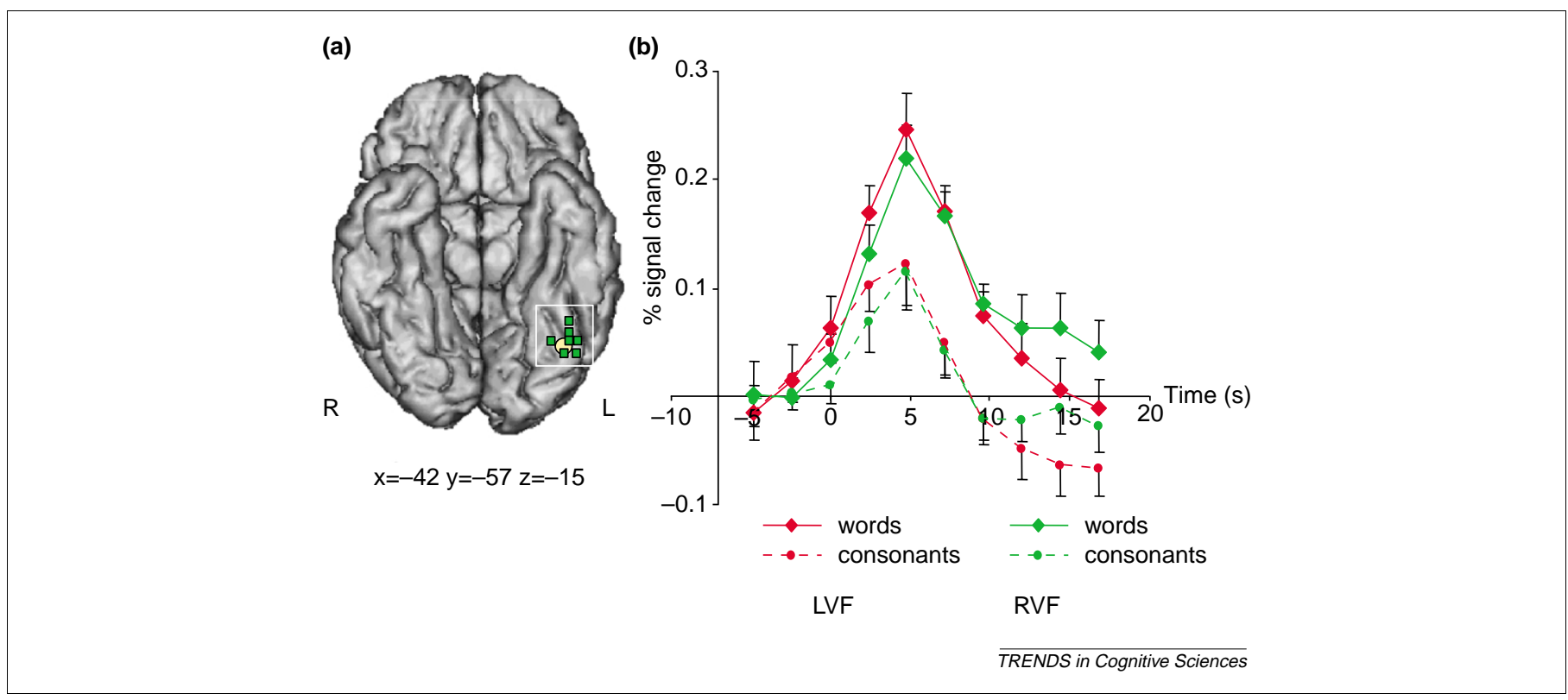

Fig. 1. (a) Peak of the Visual Word Form Area (VWFA) identified in individual subjects (green squares) and in group analyses (yellow circle) projected onto the inferior surface of a normalized brain. L indicates left hemisphere, $R$ right. (b) Percentage change in BOLD signal for words and consonant strings versus checkerboards in the left and right hemifields at the peak of the group VWFA, averaged across subjects (bars represent the intersubject standard error). Adapted from Ref. [8] by permission of Oxford University Press. 


\section{Box 1. Neuropsychological evidence}

Pure alexia (PA; also known as 'word form dyslexia' or 'letter-byletter reading') results in an impaired ability to read words in a rapid, parallel fashion $[46,47]$. The locus of damage to the visual system leading to PA coincides with the Visual Word Form Area (VWFA) (see Fig. I). The 'purity' of the syndrome refers both to the sparing of language input modalities other than vision and also to the apparent selectivity of the deficit for visual words, although the specificity of this deficit across visual domains is perhaps more a matter of relative impairment than absolute dissociation [48]. If a word is spelled-out orally or traced on the skin, PA patients can recognize it more easily than by sight. In contrast to global alexia, PA patients are able to identify single letters, and develop letter-by-letter reading strategies resulting in reading latencies that increase by hundreds of milliseconds for each additional letter [49]. Callosal lesions of fibers connecting the right-hemispheric visual regions to the VWFA provoke a subtype of PA restricted to words displayed in the left half of the visual field $[9,50]$. (a)

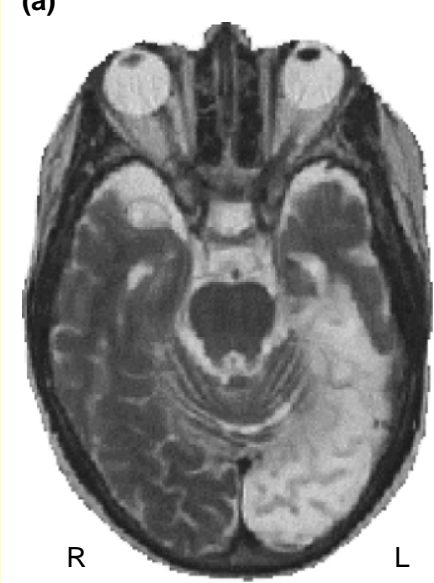

(b)

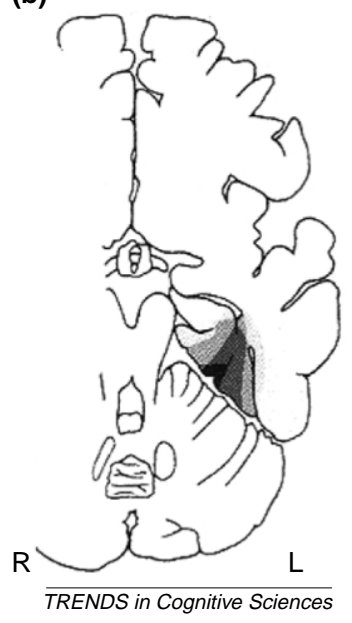

Fig. I. Extent and location of left posterior lesions are related to functional disruptions in reading. (a) An anatomical MRI of a severely alexic patient demonstrates widespread left hemisphere damage. Reproduced from [51] by permission of Psychology Press. (b) Pure alexia (letter-by-letter reading) is linked with damage to areas surrounding the Visual Word Form Area, via quantitative lesion topography studies that contrast patients with and without symptoms of pure alexia. Adapted from [47] by permission of Oxford University Press.

subliminal activation demonstrate that computations in the VWFA are largely automatic, and that the stimulusdriven responsivity of this region is at least in part dissociable from top-down influences.

\section{Stimulus-specific tuning}

The response properties of the VWFA demonstrate a form of tuning to learned letter stimuli, which produce greater activity than well-controlled pseudoletters, that can be measured by blocks of PET data [19], as well by the first $200 \mathrm{~ms}$ of activity in MEG and intracranial electrical recordings [16]. Furthermore, individual subjects' accuracy at speeded letter recognition systematically correlates with the level of activation in the VWFA [20].

\section{Spatial invariance}

Although the VWFA is left lateralized in most subjects, its response properties are relatively invariant across a range of retinal positions. Visual stimuli presented to the left or right visual hemifields follow different neural pathways (Box 2), which result in different activation patterns throughout retinotopically organized visual areas. In the VWFA, however, fMRI responses to words versus control stimuli are equivalent across both visual fields [9] (Fig. 1b). This response property has been associated with a leftlateralized ERP component recorded over left inferior temporal cortex $\sim 250 \mathrm{~ms}$ after a word is presented to the left or right visual field $[9,21]$.

\section{Invariance across visual features}

Some response characteristics of the VWFA suggest a relative insensitivity to variations in surface features of presented letters (i.e. letter case, font, size). For example, the VWFA shows equally robust fMRI responses when words (versus control stimuli) are presented in a familiar format (e.g. 'table') and in a perceptually novel mixed-case format (e.g. 'tAbLe') [10]. Furthermore, when a word is repeated immediately, fMRI and N200 ERP responses associated with the VWFA demonstrate sensitivity to word repetition that is independent of changes in letter case $[18,21]$. Case invariance holds even for upper and lowercase letters that share no visual similarity but are related only by arbitrary cultural convention (e.g. a and A, g and G) $[22,23]$. This indicates that the abstract processes of the VWFA go beyond size and shape normalization and have become attuned to the specific demands of reading.

\section{Sensitivity to orthographic regularity}

The VWFA is generally insensitive to factors that influence lexical access, such as contrasts between high- and lowfrequency words [24], or between real and pseudowords, as revealed by event-related fMRI analyses [11,23,25]. However, it demonstrates a sublexical sensitivity to the orthographic regularities by which letters form words. Novel, but well-structured letter strings (i.e. pseudowords), produce more robust fMRI responses than novel but poorly structured letter strings [8]. Similar phenomena have been demonstrated within the first $200 \mathrm{~ms}$ of stimulus processing within ERP and MEG responses associated with the left ventrotemporal region [13,26,27].

\section{Origins of the VWFA specialization}

Taken together, the response properties of the VWFA are largely consistent with a proposed level of perceptual processing in word recognition that constructs an abstract representation of letters sequenced into word forms [28]. But how did this area of the visual system evolve to have response properties that show specific sensitivity to invariant and abstract properties of a class of stimuli that never existed before the relatively recent invention of alphabetic writing systems? In addressing this question, we draw upon the deep parallels between word form skills and more general 'perceptual expertise' effects that arise out of extensive experience with a particular domain of visual stimuli (for reviews, see [29,30].

\section{The perceptual expertise account}

The emergence of perceptual expertise in several visual domains (i.e. bird experts, car experts) has been linked to 


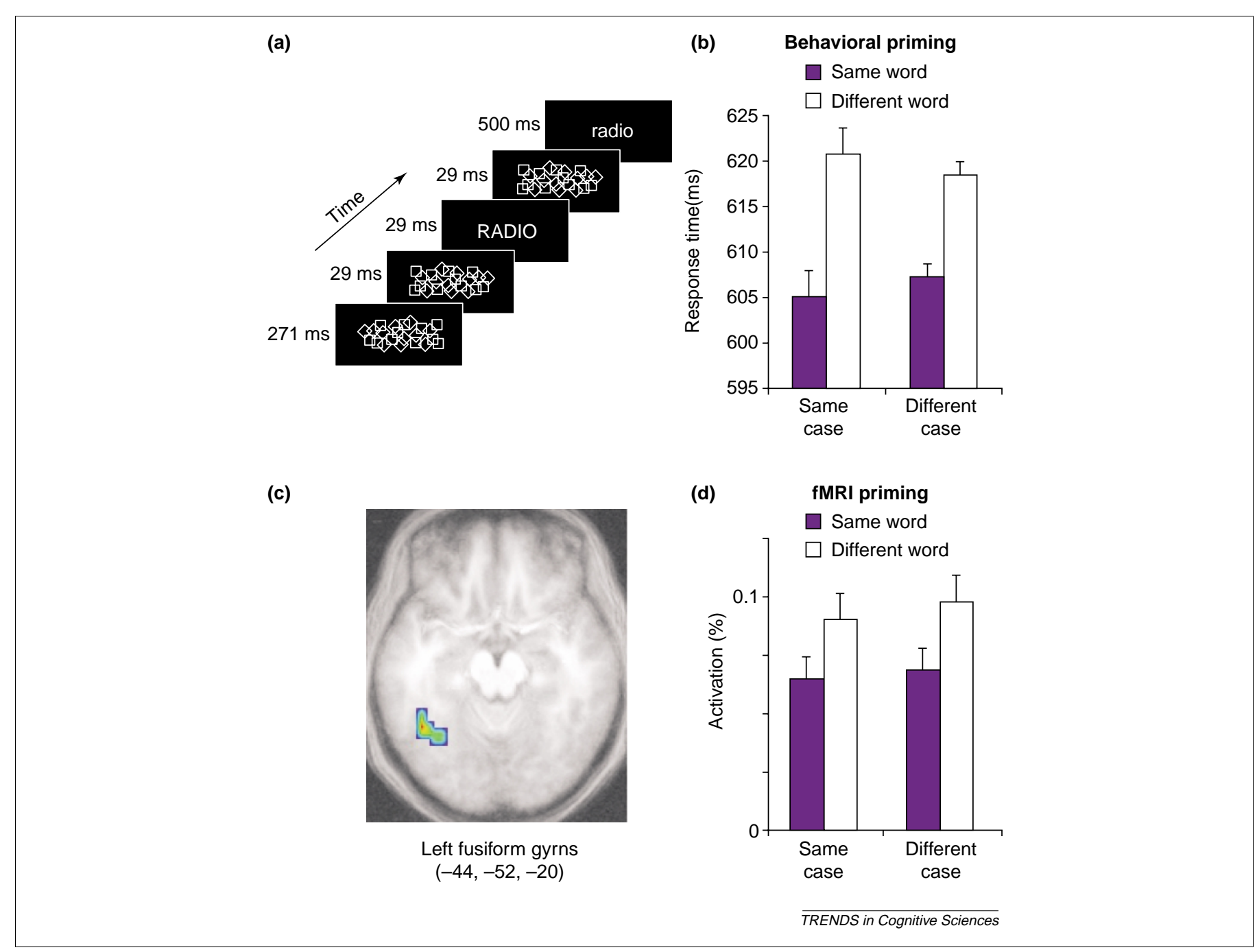

Fig. 2. Evidence for subliminal activation of a case-invariant representation of words in the left fusiform gyrus. (a) A subliminal priming paradigm allowed the presentation of a short masked prime followed by a word target. Subjects were engaged in a semantic classification task on target words and were not unaware of the presence of primes. (b) The behavioral results indicated a reduction of response time when the same word was repeated as prime and as target, irrespective of case change. (c) The cerebral bases of this repetition priming were identified by searching the whole brain for regions of reduced activation on repeated-word trials. This revealed the visual word form area in the left fusiform gyrus. (d) The activation profile of this area, relative to control trials with masks only, parallels response times in showing reduced activation on repeated trials irrespective of case change. This suggests that this area holds a case-invariant neural code for visual words and that this code can be activated automatically without awareness. Adapted from Ref. [18] by permission of Nature Publishing Group.

enhanced perception of category members via an increased ability to integrate information across the entire stimulus [31]. These processing benefits are linked to functional reorganizations in the response properties of a region of the right fusiform gyrus [32], which are also associated with changes in the response properties of ERPs recorded within the first $200 \mathrm{~ms}$ of processing stimuli [33]. Such perceptual expertise effects demonstrate principled ways in which experience can influence the functional organization of posterior-perceptual regions of the visual system.

We propose here that the VWFA constitutes a special case of perceptual expertise, in which extensive visual experience with a class of stimuli drives enhancement of perceptual mechanisms and changes in the supporting functional architecture in the left fusiform gyrus (see discussion on laterality issues below). This view on the origin of the VWFA implies that the specialization process should be protracted over a long period of experience and that the response properties of the VWFA should evolve in parallel with the rise of cognitive hallmarks of expertise. It also predicts that, over the course of development, individual differences in VWFA activity should be more closely tied to expertise than to maturation.

\section{Development of the VWFA}

Behavioral evidence on word perception supports the case for protracted development of perceptual expertise. Unlike adults, 6- to 7-year-old children (American first-graders) demonstrate robust word-length effects, which gradually diminish over the following years but are still detectable as late as grade 5 (11-12 years old) [34]. Although enhanced abilities to perceive well-structured letter strings appear early in the reading process, such effects are restricted to high-frequency words and do not generalize to novel strings until 10-13 years of age [4] (although see [35]). Developmental studies of the N200 ERP responses to letter strings demonstrate a similar pattern, suggesting that 10-year-olds produce adult-like responses to highfrequency words but fail to generalize such responses to pseudowords [21]. 


\section{Box 2. Model of functional anatomy for invariant word perception}

\begin{abstract}
We propose a simple anatomical and functional model of the visual stages of word reading, which follows the general principles that govern object recognition in the visual system of primates (Fig. I). Separate pathways in left and right hemispheres are integrated in the left lateralized Visual Word Form Area (VWFA), which mediates between visually specific input, and more abstract linguistic areas responsible for lexical, semantic and phonological processes. Although the precise

phonological processes are currently less clearly defined, functional areas probably include the left angular gyrus [52], left inferior frontal cortex [53], and temporal regions anterior to the VWFA [54]. Finally, ventral visual regions receive top-down attentional influences associated with left and right parietal regions that are likely to affect all processing levels, and whose impairment might therefore lead to various forms of neglect dyslexia.
\end{abstract} projections from VWFA to systems involved in lexical, semantic and

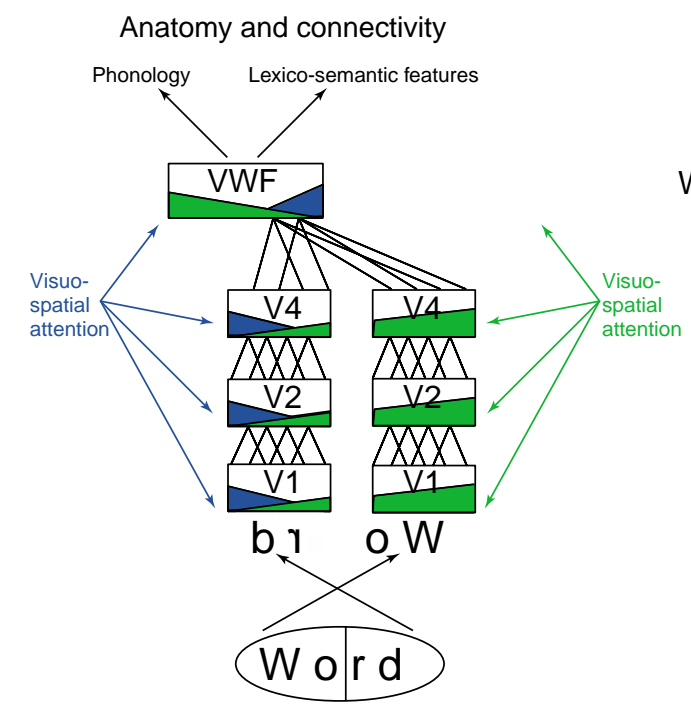

Functional properties

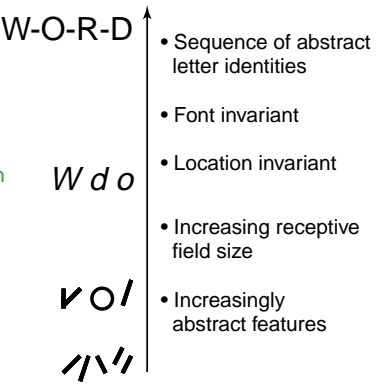

TRENDS in Cognitive Sciences

Fig. I. A tentative model of functional anatomical pathways involved in visual perception of words. Letter strings are first processed in ventral occipital regions (V1 to V4) contralateral to the stimuli, building up increasingly abstract visual representations (right). For stimuli in the left visual field, information is conveyed from the right to the left hemisphere through fiber tracts in the splenium of the corpus callosum and over the posterior horns of the lateral ventricles [50]. This right hemisphere mediated pathway and the direct left hemisphere pathway eventually converge in a structure within the left-hemispheric fusiform gyrus (the VWFA), where retinotopic coding is lost. The two pathways can be differentially modulated by visuo-spatial attention associated with input from left (blue) and right (green) parietal areas.

The progressive development of the VWFA seems closely tied to the progression of skill, rather than being merely a matter of maturation. Shaywitz and colleagues [36] examined children's fMRI responses across a range of ages ( $7-18$ years) and reading abilities (impaired to highly skilled). Activation levels of the VWFA and other nearby regions were positively correlated with standardized scores in grapheme-phoneme decoding ability, even when the effects of age were taken into account. Such findings suggest that successful mastery of graphemephoneme conversion (i.e. decoding) is a critical precursor to the development of the adult-like response properties of the VWFA. Behavioral evidence supports a similar link between decoding and enhancements of word recognition [37]. Furthermore, training effects in reading-impaired children have recently been linked to changes in fMRI activation, including posterior occipitotemporal regions in the vicinity of the VWFA [38], as children attempt to link letters to sounds.

\section{Conclusions and future directions}

We started this article with a paradox: how can reading - a recent cultural invention - rely on a cerebral substrate that is tuned to the abstract properties of a class of stimuli that did not exist for most of human evolution? The hypothesis of a progressive specialization of the left VWFA over the course of reading acquisition avoids problems inherent to the notion of a ready-made 'word recognition module'. The similarity between reading and other forms of acquired visual expertise emphasizes that our visual system progressively adapts to the tasks to which we put it. Understanding this process of progressive adaptation requires the study of the interplay between the rise of perceptual expertise during skill development and the associated changes in cortical function. We point out the presence of convergent evidence that links the psychological effects that are critical to expert-level visual word form perception with the response properties of a particular cortical region that can be systematically identified and probed.

Establishing a link between structure and function also raises additional questions of a kind that were not previously possible, because they are inherently products of the particular structure involved. For example, why does this particular region become reliably specialized in the same place for most individuals, and what pre-existing properties of this region predispose it for this specific specialization?

We propose that future work in this area should involve mapping the genetically determined properties of the 


\title{
Box 3. VWFA in developmental dyslexia
}

\begin{abstract}
Developmental dyslexia is a syndrome impacting $\sim 5 \%$ of the population worldwide, producing impairments in learning to read that are commonly linked to phonological language difficulties. Adults with a history of developmental dyslexia demonstrate a reduced tendency to activate VWFA (see Fig. I), among other regions, in

response to visual words and pseudowords as measured by $\mathrm{fMRI}$ $[44,45]$ and MEG responses within the first $200 \mathrm{~ms}$ [14]. Such differences have not yet been associated with neuropathology in this region, but may instead reflect the absence of a specialization that accrues over the course of years of successful reading experience [55].
\end{abstract}

(a)

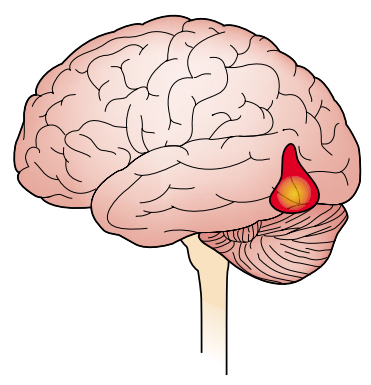

(b)

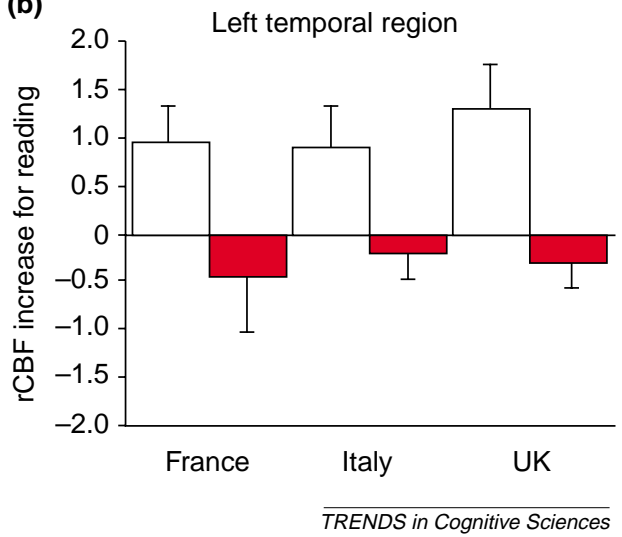

Fig. I. (a) Area consistently more active for normal readers versus dyslexic adults during reading tasks, with a peak difference located near the VWFA ( $x=-52$, $y=-60, z=-14)$. Activations displayed for this analysis are restricted to areas that consistently demonstrated group differences between normal and dyslexic adults under conditions that included both explicit and implicit reading tasks, and subject populations reading French, Italian, and English. (b) Bar graphs illustrate the PET measures of increased relative cerebral blood flow in this region for each language. Error bars indicate 1 standard error. Normal adult readers showed an increase in rCBF (white bars), whereas dyslexic readers (red bars) consistently failed to show this response. Redrawn with permission from Ref. [44]. (C) 2001 American Association for the Advancement of Science.

primate ventral visual pathway and linking these to the perceptual processing demands of visual word forms. For example, inferotemporal (IT) cortex is well known for its role in invariant object recognition. IT neurons have vast receptive fields and respond selectively to certain objects or parts of objects across large changes in size, position and illumination $[39,40]$. They also exhibit learning phenomena, even for essentially arbitrary figures [41]. Furthermore, combined optical imaging and single-cell recordings suggest that the IT cortex is composed of a mosaic of neuronal patches that respond to elementary shapes, whose combination can encode arbitrary objects [39,42]. Remarkably, some of those primitive shapes closely resemble printed or handwritten characters, such as $\mathrm{T}$ junctions, a figure-of-eight, a star, and so forth [39]. These shapes must have been selected, in the course of phylogenetic and/or ontogenetic evolution, for their ability to provide a combinatorial code capable of representing many different objects. The capability of part of this region to become responsive to learned letters is thus no accident but reflects the evolutionary history of the visual system combined with experience-dependent adaptations.

The association of visual words with a precise subregion of the fusiform gyrus can also tentatively be explained by the prior structure of the visual system. Two gradients of selectivity that cut across all areas of the ventral visual system have been identified [43]. Sensitivity to increasingly larger visual units varies along a posterior-toanterior gradient, whereas sensitivity to increasingly eccentric sectors of the retina varies along a lateral-tomesial gradient. Strikingly, the regions that show particular responsivity to objects, faces, or houses occupy reproducible locations within those gradients, corresponding to the differential requirements that they put on peripheral or foveal vision [43]. Similarly, we propose that the VWFA occupies a reproducible location on these gradients because this region best meets dual requirements of fine foveal discrimination and invariant recognition of letter- and wordsize units. One novel prediction of this hypothesis is that the precise location of the VWFA could be systematically predicted in prereading children on the basis of responses to more primitive visual mapping stimuli.

In closing, we argue that the emergence of expertise in visual word recognition can be understood as a minimal reconfiguration of the visual system to meet the specific requirements of expert reading. Much of the ventral processing stream is already well adapted to the invariant recognition of fine basic shapes in the fovea. Over the course of years of extensive experience in extracting invariant information from visual word stimuli, perceptual processes are progressively reorganized, but only in those who become skilled readers. Research with dyslexic adults indicates that, among other areas, the VWFA fails to increase its activity in response to word forms [44,45] (Box 3). A basic functional anatomical model of the typical development of this specialization might provide a framework for the investigation and treatment of atypical development, as in the case of developmental dyslexia.

\section{References}

1 Rayner, K. and Pollatsek, A. (1989) The Psychology of Reading, Prentice-Hall

2 Nazir, T.A. et al. (1998) Letter legibility and visual word recognition. Mem. Cogn. 26, 810-821 
3 Reicher, G.M. (1969) Perceptual recognition as a function of meaninfulness of stimulus material. J. Exp. Psychol. 81, 275-280

4 Gibson, E.J. et al. (1962) The role of grapheme-phoneme correspondence in the perception of words. Am. J. Psychol. 75, 554-570

5 Carr, T.H. et al. (1979) Orthography and familiarity effects in word processing. J. Exp. Psychol. Gen. 108, 389-414

6 Haxby, J.V. et al. (2000) The distributed human neural system for face perception. Trends Cogn. Sci. 4, 223-233

7 Fiez, J.A. and Petersen, S.E. (1998) Neuroimaging studies of word reading. Proc. Natl. Acad. Sci. U. S. A. 95, 914-921

8 Cohen, L. et al. (2002) Language-specific tuning of visual cortex? Functional properties of the visual word form area. Brain 125, 1054-1069

9 Cohen, L. et al. (2000) The visual word form area: spatial and temporal characterization of an initial stage of reading in normal subjects and posterior split-brain patients. Brain 123, 291-307

10 Polk, T.A. and Farah, M.J. (2002) Functional MRI evidence for an abstract, not perceptual, word-form area. J. Exp. Psychol. Gen. 131, 65-72

11 Dehaene, S. et al. (2002) The visual word form area: a prelexical representation of visual words in the fusiform gyrus. Neuroreport 13 , 321-325

12 Bentin, S. et al. (1999) ERP manifestations of processing printed words at different psycholinguistic levels: time course and scalp distribution. J. Cogn. Neurosci. 11, 235-260

13 McCandliss, B.D. et al. (1997) Brain plasticity in learning visual words. Cogn. Psychol. 33, 88-110

14 Helenius, P. et al. (1999) Dissociation of normal feature analysis and deficient processing of letter-strings in dyslexic adults. Cereb. Cortex 9 , 476-483

15 Tarkiainen, A. et al. (2002) Dynamics of visual feature analysis and object-level processing in face versus letter-string perception. Brain $125,1125-1136$

16 Nobre, A.C. et al. (1994) Word recognition in the human inferior temporal lobe. Nature 372, 260-263

17 Büchel, C. et al. (1998) A multimodal language region in the ventral visual pathway. Nature 394, 274-277

18 Dehaene, S. et al. (2001) Cerebral mechanisms of word masking and unconscious repetition priming. Nat. Neurosci. 4, 752-758

19 Price, C.J. et al. (1996) Demonstrating the implicit processing of visually presented words and pseudowords. Cereb. Cortex 6, 62-70

20 Garrett, A.S. et al. (2000) Cortical activity related to accuracy of letter recognition. Neuroimage 11, 111-123

21 Posner, M.I. and McCandliss, B.D. (1999) Brain circuitry during reading. In Converging Methods for Understanding Reading and Dyslexia (Klein, R.M. and McMullen, P.A., eds) pp. 305-337, MIT Press

22 McCandliss, B.D. et al. (2000) Habituating cognitive codes for individual words within the visual word form area: an event related fMRI study. J. Cogn. Neurosci. Suppl., 118

23 Dehaene, S. et al. (2003) Letter binding and invariant recognition of masked words: behavioral and neuroimaging evidence. Psychol. Sci. (in press)

24 Fiebach, C.J. et al. (2002) fMRI evidence for dual routes to the mental lexicon in visual word recognition. J. Cogn. Neurosci. 14, 11-23

25 Beauregard, M. et al. (1997) The neural substrate for concrete, abstract, and emotional word lexica: a positron emission tomography study. J. Cogn. Neurosci. 9, 441-461

26 Ziegler, J.C. et al. (1997) Word, pseudoword and nonword processing: a multitask comparison using event-related-potentials. J. Cogn. Neurosci. 9, 758-775

27 Breier, J.I. et al. (1998) Relative timing of neuronal activity in distinct temporal lobe areas during a recognition memory task for words. J. Clin. Exp. Neuropsychol. 20, 782-790

28 Carr, T.H. and Posner, M.I. (1995) The impact of learning to read on the functional anatomy of language processing. In Language and Literacy: Comparative Approaches (de Gelder, B. and Morais, J., eds) pp. 267-294, MIT Press

29 Tarr, M.J. and Gauthier, I. (2000) FFA: a flexible fusiform area for subordinate-level visual processing automatized by expertise. Nat. Neurosci. 3, 764-769
30 Tarr, M.J. and Cheng, Y.D. (2003) Learning to see faces and objects. Trends Cogn. Sci. 7, 23-30

31 Rossion, B. et al. (2000) The N170 occipito-temporal component is delayed and enhanced to inverted faces but not to inverted objects: an electrophysiological account of face-specific processes in the human brain. Neuroreport 11, 69-74

32 Gauthier, I. et al. (2000) Expertise for cars and birds recruits brain areas involved in face recognition. Nat. Neurosci. 3, 191-197

33 Tanaka, J.W. and Curran, T. (2001) A neural basis for expert object recognition. Psychol. Sci. 12, 43-47

34 Aghababian, V. and Nazir, T.A. (2000) Developing normal reading skills: aspects of the visual processes underlying word recognition. J. Exp. Child Psychol. 76, 123-150

35 Chase, C.H. and Tallal, P. (1990) A developmental, interactive activation model of the word superiority effect. J. Exp. Child Psychol. $49,448-487$

36 Shaywitz, B.A. et al. (2002) Disruption of posterior brain systems for reading in children with developmental dyslexia. Biol. Psychiatry 52, $101-110$

37 Share, D.L. (1999) Phonological recoding and orthographic learning: a direct test of the self-teaching hypothesis. J. Exp. Child Psychol. 72, 95-129

38 Temple, E. (2003) Neural deficits in children with dyslexia ameliorated by behavioral remediation: evidence from functional fMRI. Proc. Natl. Acad. Sci. U. S. A. 100, 2860-2865

39 Tanaka, K. (2003) Columns for complex visual object features in inferotemporal cortex: clustering of cells with similar but slightly different stimulus selectivities. Cereb. Cortex 13, 90-99

40 Einhauser, W. et al. (2002) Learning the invariance properties of complex cells from their responses to natural stimuli. Eur. J. Neurosci. $15,475-486$

41 Logothetis, N. (2000) Object recognition: holistic representations in the monkey brain. Spat. Vis. 13, 165-178

42 Tsunoda, K. et al. (2001) Complex objects are represented in macaque inferotemporal cortex by the combination of feature columns. Nat. Neurosci. 4, 832-838

43 Malach, R. et al. (2002) The topography of high-order human object areas. Trends Cogn. Sci. 6, 176-184

44 Paulesu, E. et al. (2001) Dyslexia: cultural diversity and biological unity. Science $291,2165-2167$

45 Shaywitz, S.E. et al. (1998) Functional disruption in the organization of the brain for reading in dyslexia. Proc. Natl. Acad. Sci. U. S. A. 95, 2636-2641

46 Warrington, E.K. and Shallice, T. (1980) Word-form dyslexia. Brain $103,99-112$

47 Binder, J.R. and Mohr, J.P. (1992) The topography of callosal reading pathways. a case-control analysis. Brain 115, 1807-1826

48 Behrmann, M. et al. (1998) Visual complexity in letter-by-letter reading: 'pure' alexia is not pure. Neuropsychologia 36, 1115-1132

49 Cohen, L. et al. (2003) Visual word recognition in the left and right hemispheres: anatomical and functional correlates of peripheral alexias. Cereb. Cortex (in press)

50 Molko, N. et al. (2002) Visualizing the neural bases of a disconnection syndrome with diffusion tensor imaging. J. Cogn. Neurosci. 14, 629-636

51 Cohen, L. and Dehaene, S. (2000) Calculating without reading: unsuspected residual abilities in pure alexia. Cogn. Neuropsychol. $17,563-583$

52 Horwitz, B. et al. (1998) Functional connectivity of the angular gyrus in normal reading and dyslexia. Proc. Natl. Acad. Sci. U. S. A. 95, 8939-8944

53 Bokde, A.L. et al. (2001) Functional interactions of the inferior frontal cortex during the processing of words and word-like stimuli. Neuron $30,609-617$

54 Giraud, A.L. and Price, C.J. (2001) The constraints functional neuroanatomy places on classical models of auditory word processing. J. Cogn. Neurosci. 12, 754-765

55 McCandliss, B.D. and Noble, K. (2003) The development of reading impairment: a cognitive neuroscience model. Mental Retard. Dev. Disab. Res. Rev. (in press) 